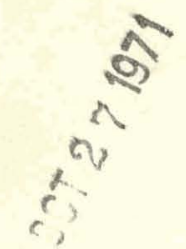

W. A. Glass, L. H. Toburen, W. E. Wilson, W. C. Roesch

* This paper is based on work performed under United States Atomic Energy Commission Contract AT(45-1)-1830 and the Pacific Northwest Laboratories of the Battelle Memorial Institute. 


\section{DISCLAIMER}

This report was prepared as an account of work sponsored by an agency of the United States Government. Neither the United States Government nor any agency Thereof, nor any of their employees, makes any warranty, express or implied, or assumes any legal liability or responsibility for the accuracy, completeness, or usefulness of any information, apparatus, product, or process disclosed, or represents that its use would not infringe privately owned rights. Reference herein to any specific commercial product, process, or service by trade name, trademark, manufacturer, or otherwise does not necessarily constitute or imply its endorsement, recommendation, or favoring by the United States Government or any agency thereof. The views and opinions of authors expressed herein do not necessarily state or reflect those of the United States Government or any agency thereof. 


\section{DISCLAIMER}

Portions of this document may be illegible in electronic image products. Images are produced from the best available original document. 


\title{
ENERGY EJECTED IN FAST PROTON COLLISIONS*
}

W. A. Giass, L. H. Toburen, W. E. Wilson, W. C. Roesch

Battelle

Pacific Northwest Laboratories

Richland, Washington

\begin{abstract}
An analysis of the relative importance of various processes by which the energy of fast protons is transferred to a medium of low atomic number shows that, in the proton energy range $300 \mathrm{keV}$ to $1700 \mathrm{keV}$, about 50 to 60 percent appears as kinetic energy of the secondary erectrons and 25 to 30 percent is involved in overcoming the binding energy of these electrons. The remaining 10 to 20 percent can be accounted for as primary excitation, recoil, etc. In this paper distributions in angle and in the kinetic energy associated with electrons ejected by protons in passing through various gas absorbers will be presented. These distributions are derived from experimentally measured cross sections. Effects of chemical binding energy on electron ejection cross sections will be considered. Distributions of secondary electrons in energy will be compared with results obtained using a binary encounter theory to illustrate the degree of applicability of this semi-classical treatment. The importance of secondary electrons for energy deposition in small sites will be considered.
\end{abstract}

* This paper is based on work performed under United States Atomic Energy Commission Contract AT(45-1)-1830 and the Pacific Northwest Laboratories of the Battelle Memorial Institute. 


\section{ENERGY EJECTED IN FAST PROTON COLLISIONS}

\section{INTRODUCTION}

In order to understand the nature of energy deposition in small volumes traversed by protons, it is essential to know the probabilities involved for energy transfer in individual collisions between the proton and the absorber molecules. In a broad sense these collisions can be classified into two categories-those that result in ionization and those that result only in excitation. This paper will be concerned with the energy and angular distributions of the secondary electrons ejected in ionizing collisions.

The experimental measurement of secondary electron $(\delta-r a y)$ spectra was partially motivated by the observation that distributions of energy deposited in small sites by protons were greatly affected by energy transport associated with $\delta$-rays. (1) The moments of these distributions do not conform to those expected in the absence of energy transport. For example, if one considers the statistical distribution of energy lost by monoenergetic protons as they traverse a short segment of path, it can be shown that the quotient of the variance by the mean of this distribution is independent of the path length. (2) This quotient, referred to as $\delta_{2}$ in the preceeding reference is expected to be approximately equal to $E_{\max } / 2 \ln \left(E_{\max } / I\right)$ where $E_{\max }$ is the maximum energy transfer to a $\delta$-ray and $I$ is the mean excitation energy of the absorber.

Quotients of the variance by the mean of ionization distributions measured in a 0.25 micron site were found to be nearly independent of proton energy for $1.7,3.0$, and $4.0 \mathrm{MeV}$ protons. The quotient was about 0.4 keV as compared with approximate values of $\delta_{2}$ calculated from energy loss straggling theory of $0.45,0.70$, and $0.88 \mathrm{keV}$ for the respective proton energies. The maximum range for the secondary electrons from 
a 1.7 MeV proton is about 1 micron, only four times the diameter of the site. Most of the secondary electrons have much shorter ranges and are absorbed within the site. Thus, it would be expected that in this case the observed value of the quotient, about $0.4 \mathrm{keV}$, be only slightly less than the $0.45 \mathrm{keV}$ predicted neglecting transport effects. The ranges of electrons for higher energy protons are substantially greater; transport effects should be relatively larger and, as a consequence, the quotient smaller than for energy loss straggling. The surprising thing is that the shape of the energy deposition distribution, as judged by the quotient of variance by mean, is so nearly independent of the proton energy. Other tests of distribution shape based on Fourier transform techniques are more sensitive. than the moments quotient. These tests confirm that indeed the distributions, hence the energy absorbed in the site from individual collisions, were nearly independent of proton energy over the range studied. This behavior indicates that transport effects have a dominant influence on the energy deposition from single collisions for protons having sufficient energy to produce secondary electrons with maximum ranges of only a few times the diameter of the absorbing site.

To better understand these transport effects and to improve our general understanding of the energy deposition problem, we have engaged in rather extensive measurements of electron emission from molecules of low atomic number when bombarded with protons in the energy range of $300 \mathrm{keV}$ to $2000 \mathrm{keV}$. The measurements have involved elemental gases such as hydrogen and nitrogen and also various hydrocarbon gases. We will present representative data showing effects of the atomic configuration on the energy and angular distribution of electrons ejected in the primary ionizing collisions; effects which may be attributed to molecular binding energies for various hydrocarbons will also be shown. 


\section{RELATIVE IMPORTANCE OF VARIOUS PRIMARY PROCESSES}

Before discussing the secondary electrons produced in ionizing collisions it is desirable to investigate their relative importance in the stopping of protons. Perhaps this can best be done by a comparison of the type shown in Fig. 1 in which 1500 keV protons on hydrogen is chosen as an example. In this figure the cross section, i.e., probability of ejection of an electron of energy, $\varepsilon$, is multiplied by the appropriate. factor so that equal areas under the curves represent equal energies transferred or equal contributions to the stopping power. The ionization potential for molecular hydrogen, I, has been set at 15.8 eV corresponding to a vertical transition to the ionized molecule. The value assigned to $I$ is somewhat ambiguous even for the hydrogen molecule and is eyen more so for more complex atomic and molecular structures. $(3,4)$ The solid lines are derived directly from experimental measurements. The area under the curve labeled $(I+\varepsilon) \varepsilon \sigma(\varepsilon)$ represents that energy first appearing in the medium as kinetic energy of secondary electrons. This represents about $55 \%$ of the stopping power of hydrogen for $1500 \mathrm{keV}$ protons. Each ionizing collision must also provide the energy to overcome the potential of the electron in the molecule. The curve labeled $(I+\varepsilon)^{2} \sigma(\varepsilon)$ illustrates the effect of adding to the energy of the ejected electron its binding energy. This effect is, of course, most apparent for the low energy secondaries. The area between this curve and the curve for kinetic energy of the secondaries represents approximately $25 \%$ of the stopping power. Hence, for $1500 \mathrm{keV}$ protons in hydrogen, about $80 \%$ of the energy 10 ss can be accounted for by electrons ejected through primary ionizing collisions.

Many primary collisions transfer too little energy to produce ionization and those that do ionize are likely to leave the molecule in an excited or dissociative state with a small 
amount of recoil energy. These energies must account for the remaining stopping power and are indicated by a hypothetical cross section- dashed line. Little specific information is available for these cross sections (4) and the curve here is meant only to indicate their existence. The area under the dashed curve was adjusted to make the total area numerically equal to the stopping power per molecule. Note that the energy represented by the shaded area is absorbed directiy by the molecule involved in the primary collisions; whereas, the kinetic energy of the ejected electrons is attenuated in subsequent collisions.

The fraction of the stopping power associated with these various modes of energy absorption are only slightly dependent on proton energy above about $200 \mathrm{keV}$. (3) This is indicated in Fig. 2 which is taken from the preceeding reference. Below $200 \mathrm{keV}$ other energy transfer mechanisms such as a charge changing and nuclear collisions become important.

The general distribution of those electrons ejected in primary collisions can be put in perspective by considering the partition of the number of electrons and the energy associated with them above and below certain kinetic energy values. For example, Table I shows that for protons having energies between 300 and $1500 \mathrm{keV}$ the mean ejected electron energy lies between 30 and $60 \mathrm{eV}$; this value is only slightly dependent on whether the target is hydrogen or nitrogen. Nearly a constant 75 percent of the kinetic energy is associated with the 20 to 25 percent of electrons having energy greater than the mean value. Half the ejected electrons have energies below 10-15 eV - i.e., over half of the ejected electrons are incapable of further ionization. On the other hand 90-95 percent of the kinetic energy is associated with that half of the total number of electrons that have energy in excess of 10 to $15 \mathrm{ev}$. 
Half the kinetic energy transferred in primary collisions is associated with fewer than 10 percent of the electrons and this percentage rapidly decreases with increasing proton energy. Electrons with energy greater than $200 \mathrm{eV}$ (i.e., those having a range greater than $10^{-6} \mathrm{gm} / \mathrm{cm}^{2}$ ) number on 1 y 3 to 6 percent of the total, but they transport between 30 and 60 percent of the available kinetic energy. Again, only about 55 percent of the absorbed proton energy appears as kinetic energy of the secondaries, but it is this energy that may be deposited beyond the molecules that lie immediately along the proton path.

MEASUREMENT OF ENERGY AND ANGULAR DISTRIBUTIONS OF SECONDARY ELECTRONS

The experimental technique for measuring the energy and angular distributions for electrons ejected in single collisions between an incident proton and target. molecule has been described in detail in a previous publication (5). The apparatus consists of a differentially pumped target cell through which a proton beam is passed. Electrons ejected in ionizing collisions within the cell exit through a slit and are then energy analyzed by an electrostatic analyzer and counted by an electron multiplier. The gas cell pressure is maintained such that the probability of a collision occurring is about one for every one hundred incident protons. The low density assures that the observed electrons result from single collisions and also minimizes the chance of scattering the ejected electron in the target. After passing through the target, the protons are collected in a Faraday cup, the output of which is used in calculating absolute values for the probability per target molecule for electron ejection in a direction, $\theta$, with an energy, $\varepsilon$; $i . e$. , the doubly differential cross section $\sigma(\varepsilon, \theta)$. The electron energy is determined with an electrostatic analyzer of the cylindrical mirror type collimated to an acceptance angle of about five degrees. 
Results shown in Fig. 3 are typical of the doubly differential cross sections. These energy spectra of ejected electrons for angles between 20 degrees and 130 degrees with respect to the proton direction were obtained for $1500 \mathrm{keV}$ protons on a hydrogen target. A broad peak is observed in the cross section near that energy at which the free electron model would predict a $\delta$-function containing all the probability for ejection in that direction. This broad distribution and the very large increase in the cross section for low energy electrons are entirely a reflection of the fact that the collisions involve electrons bound in atoms and molecules. It is somewhat remarkable that the free electron model, which has been the basis for so many theories regarding straggling and energy loss; has been so successful. It is obviously a gross oversimplification of the existing situation, particularly where the angular distribution is concerned.

ANGULAR DISTRIBUTION OF $\delta$-RAY ENERGY

In considering energy deposition and energy transport by $\delta$-rays, the proton path can be considered a straight line along which electrons originate with certain probability distributions in both energy and angle. Representative distributions of this kinetic energy of ejected electrons are displayed in Fig. 4 and 5 as polar plots. The radial coordinate, $\rho$, represcnts the quantity defined by:

$$
\rho=\int_{\varepsilon_{0}}^{\infty} \varepsilon \sigma(\varepsilon, \theta) d \varepsilon .
$$

That is, the kinetic energy initially appearing in the medium as clectrons ejected in an angular interval de about an angle $\theta$ with energy greater than $\varepsilon_{0}$ is rcpresented by the magnitude of $\rho$. This representation sacrifices detailed information regarding the distribution of electron energies at a particular angle in order to plot the energy transfer in two dimensions. 
Two values of $\varepsilon_{0}$ were chosen: one to indicate the total kinetic energy transfer and the other to indicate that which may be deposited at distances greater than $10^{-6} \mathrm{gm} / \mathrm{cm}^{2}$ from the proton path.

The distributions are noticeably altered by considering only the more energetic electrons. This change in shape is most apparent for the lower energy protons. Electrons ejected by $300 \mathrm{keV}$ protons have a maximum energy of about $650 \mathrm{eV}$ and most have energies less than $200 \mathrm{eV}$. But, the three percent that have energy in excess of $200 \mathrm{eV}$ carry about one third the kinetic energy. The most probable angle at which this energy is ejected is about $30^{\circ}$ as compared to 40 to 50 degrees, when electrons of all energies are considered. At higher incident proton energies the binding energy is of less consequence. The distributions of energy become more concentrated in a conical sector which narrows and opens more nearly normal to the proton path as the proton energy increases. This trend can be seen clearly in Fig. 4 .

Nitrogen presents a target molecule quite different from hydrogen. The five outer electrons in each atom are bound with energy similar to the electrons in hydrogen, but each atom also has two electrons bound with about $400 \mathrm{eV}$. The energy transfer cross sections $[\varepsilon \sigma(\varepsilon, \theta)]$ are, when integrated, from 5 to 7 times 1 arger than for hydrogen and they show a considerably different angular distribution of ejected electron energy. Fig. 5 illustrates the effect on the angular distri.. bution of kinetic energy produced by the more complex atomic structure. There is a gencral trend fur the energy to be transferred in a more forward direction which is a reflection of the higher electronic binding energy. Effects due to K-shell electrons are most evident in distributions for more energetic protons; $300 \mathrm{keV}$ protons have a small probability of interacting with electrons bound with $400 \mathrm{eV}$ energy. The peak at about 
60 degrees in the measured distribution for 1700 keV incident protons is due to ejection of the loosely bound valence electrons. The angle is not greatly different from that for hydrogen and the magnitude is 4 to 5 times greater; this shows the similar behavior of the valence electrons to those of hydrogen. The peak at about 35 degrees can be attributed to. $\mathrm{K}$-shell electrons. Although the collision cross section is smaller than for the 60 degree peak the energy associated with the ejected electrons is greater and the result is a larger energy transfer. Significant energy transfer into angles greater than 90 degrees is also noted for nitrogen. This energy is associated with Auger electrons and resonant collisions which result in a more isotropic energy transfer.

\section{ENERGY DISTRIBUTIONS OF EJECTED ELECTRONS}

A first approximation to the energy distribution can be obtained by considering the medium as a free electron gas. This model predicts the familiar scattered electron energy distribution with a cross section proportional to $1 / \varepsilon^{2}$. A convenient way to observe deviations from this model is to plot the measured cross sections on an ordinate scale of $\varepsilon^{2} \sigma(\varepsilon)$ vs. the logarithm of electron energy. On this scale, cross sections from the free electron model become horizontal lines with magnitude calculated from classical Rutherford scattering. Also in this type of plot equal areas under different segments of the curve represent equal amounts of energy transferred to electrons in corresponding energy intervals.

Electron energy distributions for 300 and 1500 keV protons on hydrogen gas are shown as solid lines in Fig. 6. The tick marks show the magnitude of the ordinate of the $1 / \varepsilon^{2}$ distribution and the arrows indicate the maximum energy that can be transferred to a free electron. For 1500 keV protons, the measured electron spectra (the solid line) agree well with 
the $1 / \varepsilon^{2}$ distribution for electrons with energy greater than a few times the molecular binding energy. Spectra for electrons ejected by $300 \mathrm{keV}$ protons show no region of real agreement. The dotted lines, however, which are from cross sections calculated on the basis of a semi-classical model show general agreement over the entire spectrum. The semiclassical treatment used in these calculations is described in detail by Rudd, et.al. (6) and is frequently referred to as a binary encounter model. The cross sections are calculated taking into account the relative velocities between the incident proton and a target electron. The electron is then treated as a free scattering center with a distribution of velocities which are normally derived from a Fock distribution for the target electrons. These calculations require only a nominal amount. of computer time, yet produce an energy spectrum that is in fair agreement with measured values. Such calculations do not provide angular distributions.

The binary encounter theory is less successful in predicting the energy spectra for nitrogen; a consequence of inadequate representation of the electron velocity in the molecule. Two tick marks are used in Fig. 7 to illustrate the. free electron cross sections, the lower one considers 5 electrons per atom and the upper tick mark includes the two $k$ electrons. As expected on the basis of the results for hydrogen, the higher energy portion of the measured spectra. lies between these two values. The peak at about 365 eV is the result of Auger transitions following $k$-shell ionization. Since the fluorescence yield in nitrogen is essentially zero, a K-shell ionization is nearly always followed by an Auger electron which leaves the target duubly ionized. About' 5 percent of the kinetic energy of secondaries produced by 1700 keV protons is in Auger electrons. This fraction drops to about 1 percent for $300 \mathrm{keV}$ protons. 


\section{EFFECTS DUE TO MOLECULAR BINDING ENERGIES}

In experimental microdosimetry it is tacitly assumed that a medium may be simulated, insofar as energy deposition (or ionization) is concerned, by a mixture of gases constituted in the appropriate atomic ratios. This practice is an experimental necessity, but we must be aware that the molecular structure of the component gases may play a part in the distribution of ionization in and about the path of a charged particle.

Fig. 8 shows the angular distribution of the cross section for ejection of electrons at selected energies. The solid 1 ines represent the cross sections for methane gas and ejected electron energies between 8 and $200 \mathrm{eV}$. The lower energy electrons show nearly an isotropic distribution with the more forward directions being preferred. As the electron energy being considered is increased, the cross sections, develop a distinct directional dependence with a maximum near the angle at which the classical free electron model would predict a $\delta$-function distribution. The magnitude of the cross section drops off rapidly-over two orders of magnitude in the energy range considered here. The cross sections resulting from the simulation of methane in atomic composition by mixtures of acetylene and hydrogen or benzene vapor and hydrogen are shown as open circles. For:ejected electron energies of about $50 \mathrm{eV}$ and greater (energies above 200 eV are not shown) a direct additive rule accurately predicts the electron ejection cross sections. There is a slight difference in Auger emission which we won't discuss here. Recall from Table I that 75 percent of the energy is associated with electrons having energy greater than about $50 \mathrm{ev}$; hovever, about 80 percent of the number of electrons generated in primary collisions have energy less than this. Therefore, the number of ions formed through primary interactions, 
particularly the low energy interactions, will depend significantly on molecular binding energies. Methane equivalent mixtures composed of ethane and etheylene with hydrogen show better agreement than do either of the mixtures shown in Fig. 8 .

$$
\text { If, as our data suggests, the number of low energy }
$$
electrons formed along a proton path is greatly different for different gas mixtures, then the distribution in number of ions deposited in small volumes will depend on the molecular composition. This is particularly important for the microdosimetry of very small sites where more of the collected ions (electrons) are a direct result of primary collisions; the energetic secondary electrons escape from the site. 


\section{REFERENCES}

1. W. A. Glass and W. C. Roesch, "Measurement of Ionization Distributions in Tissue-Equivalent Gas". Radiat. Res., (in press).

2. A. M. Kellerer, "Analysis of Patterns of Energy Deposition; A Survey of Theoretical Relations in Microdosimetry". In Proceedings second Symposium on Microdosimetry, pp. 107-134. Commission of the European Communities, Euratom, Brussels, (1969).

3. W. E. Wilson, "Stopping Power Partition and Mean Energy Loss for Energetic Protons in Hydrogen". Radiat. Res.., (in press).

4. R. L. Platzman, "Total Ionization in Gases by High-Energy Particles: An Appraisal of Our Understanding". Int. J. of App. Radiat. and Isotopes, 10, 116-127 (1961).

5. L. H. Toburen, "Distributions in Energy and Angle of Electrons Ejected from Molecular Nitrogen by 0.3 to 1.7 MeV Protons". Phys. Rev. A $\underline{3}, 216-228$ (1971).

6. M. E. Rudd, D. Gregoire, and J. B. Crooks, "Comparison of Experimental and Theoretical Values of Cross Section for Electron Production by Proton Impact". Pirys. Rev. A $1635-1640$, (1971). 


\section{FIGURE CAPTIONS}

Fig. 1 - Energy transfer to hydrogen gas by 1500 keV protons. Energy represented by the unshaded area first appears as kinetic energy of ejected electrons; that represented by the shaded area is deposited in the interacting molecule.

Fig. 2 - Energy partition for primary collisions of protons in hydrogen. The curve marked $F_{T}$ is the fraction appearing as kinetic energy of ejected electrons; $F_{I}$ is the fraction associated with binding energy of ejected electrons; $F_{E}$ is the fraction associated with primary excitation; $F_{C C}$ is the fraction associated with charge changing collisions.

Fig. 3 - Energy spectra for electrons ejected from hydrogen by 1500 keV proton impact plotted at representative angles.

Fig. 4 - Energy transfer to secondary electrons in hydrogen as a function of angle for electron energies greater than $200 \mathrm{eV}$ and for all electron energies.

Fig. 5 - Energy transfer to secondary electrons in nitrogen as a function of angle for electron energies greater than $200 \mathrm{eV}$ and for all electron energies.

Fig. 6 - Ejected electron energy spectrum for $300 \mathrm{keV}$ and $1500 \mathrm{keV}$ protons on hydrogen compared with the free electron model and a binary encounter model.

Fig. 7 - Ejected electron energy spectrum for $300 \mathrm{keV}$ and $1700 \mathrm{keV}$ protons on nitrogen compared to the free electron model and a binary encounter model.

Fig. 8 - Comparison of angular distributions of electrons ejected from methane by 1 MeV protons with distributions composed from acetylene and hydrogen and from benzene and hydrogen.

Table I. Partition in number and kinetic energy of ejected electrons for various electron energies. 


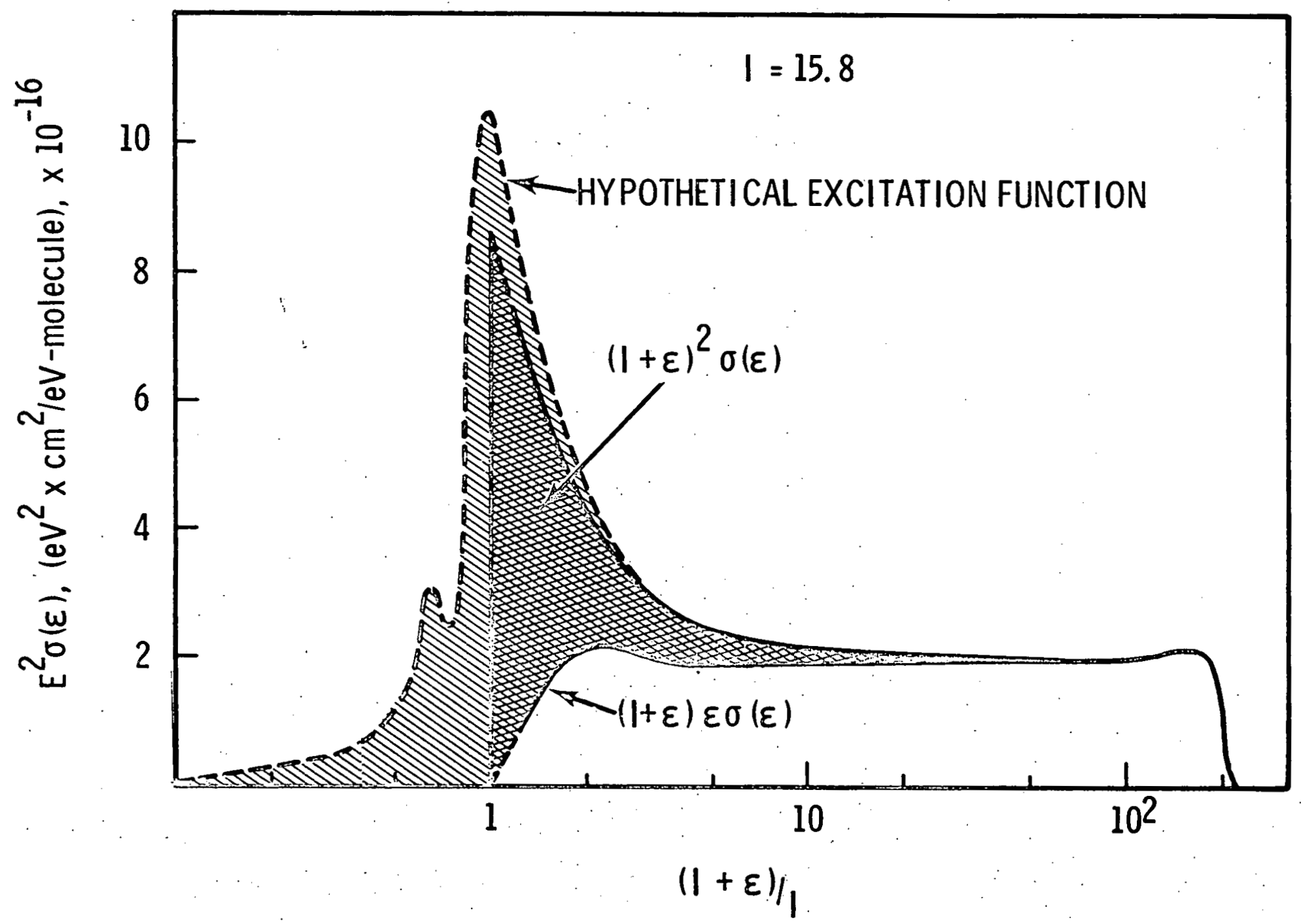

Fig. 1 


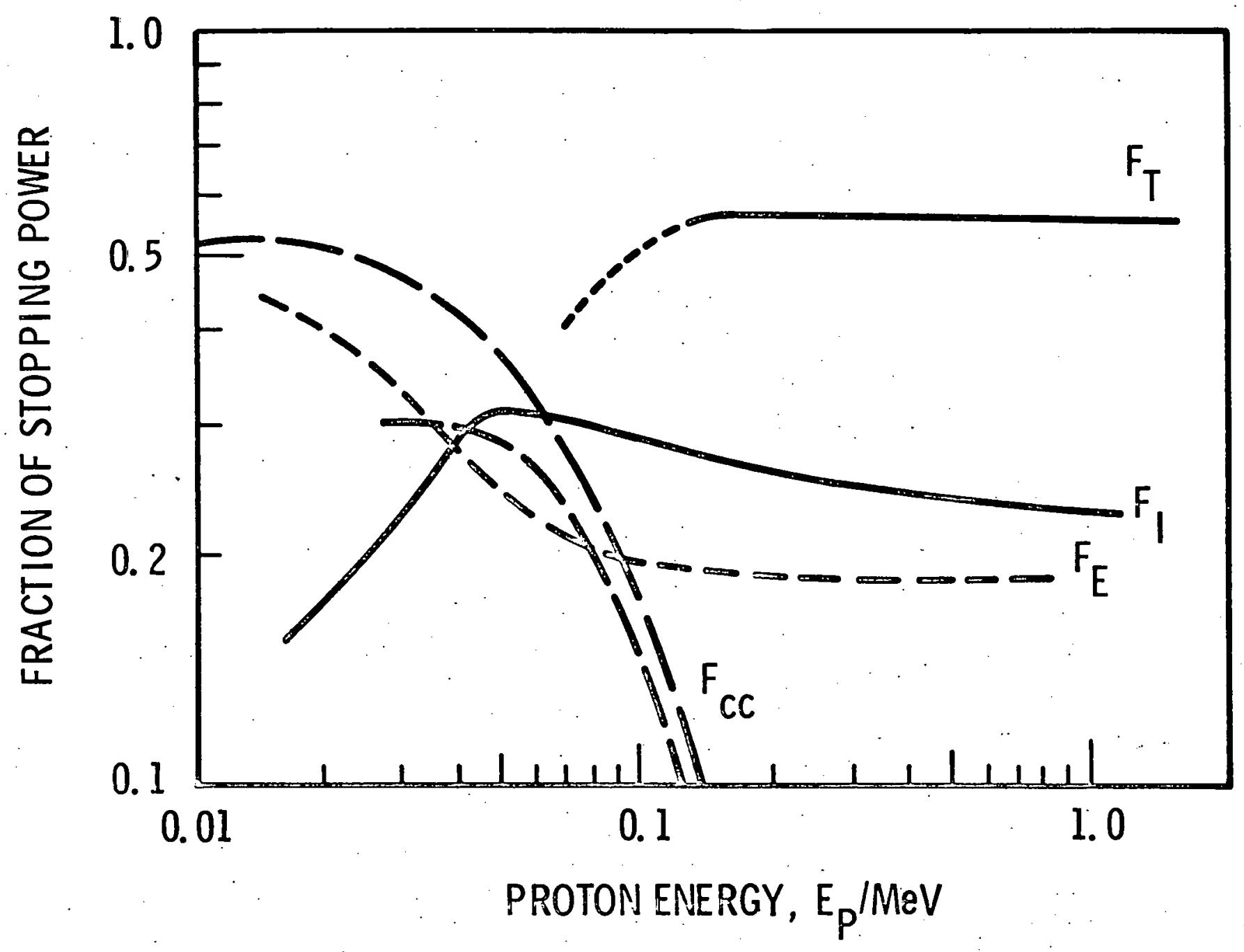

Fig. 2 


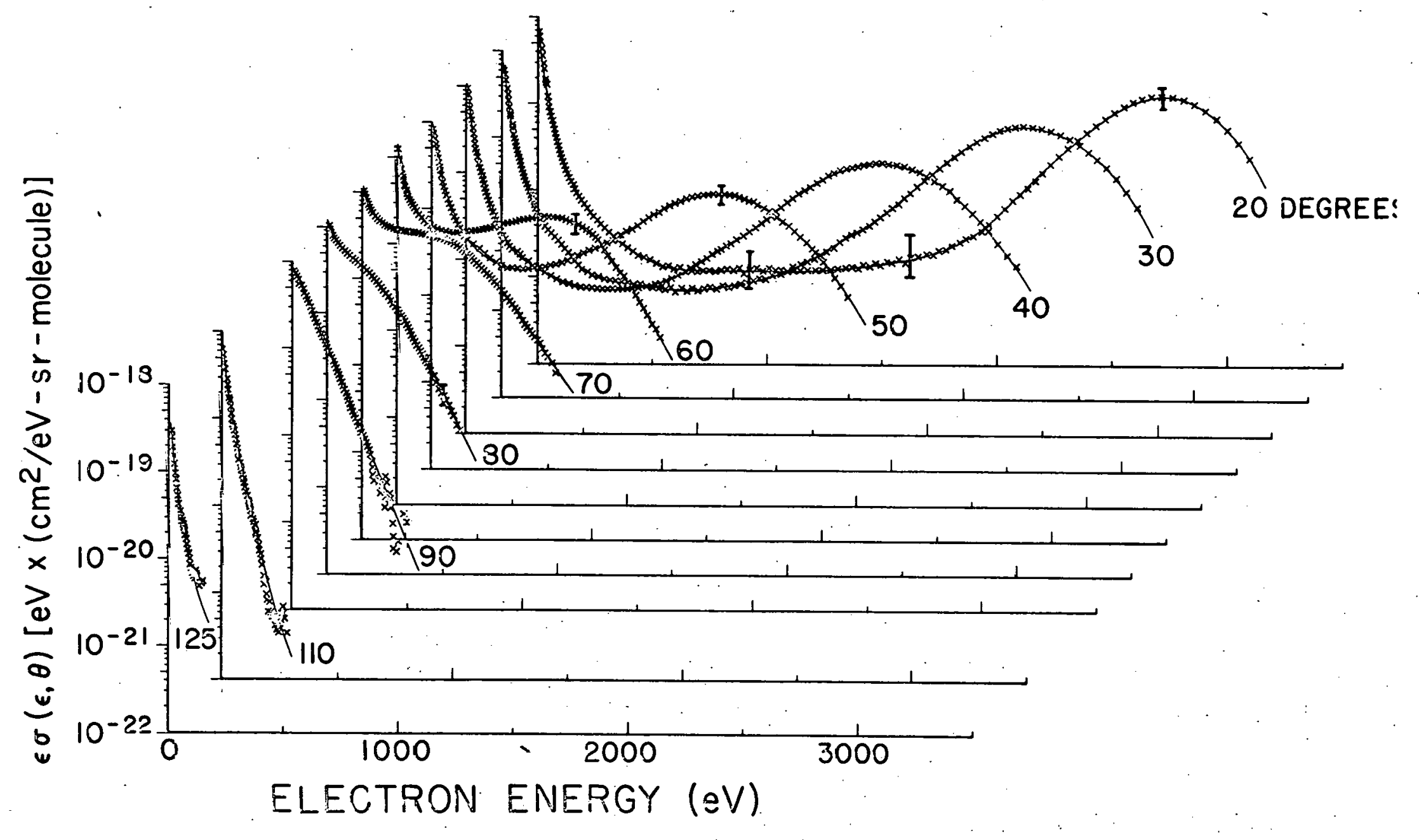

Fig. 3 

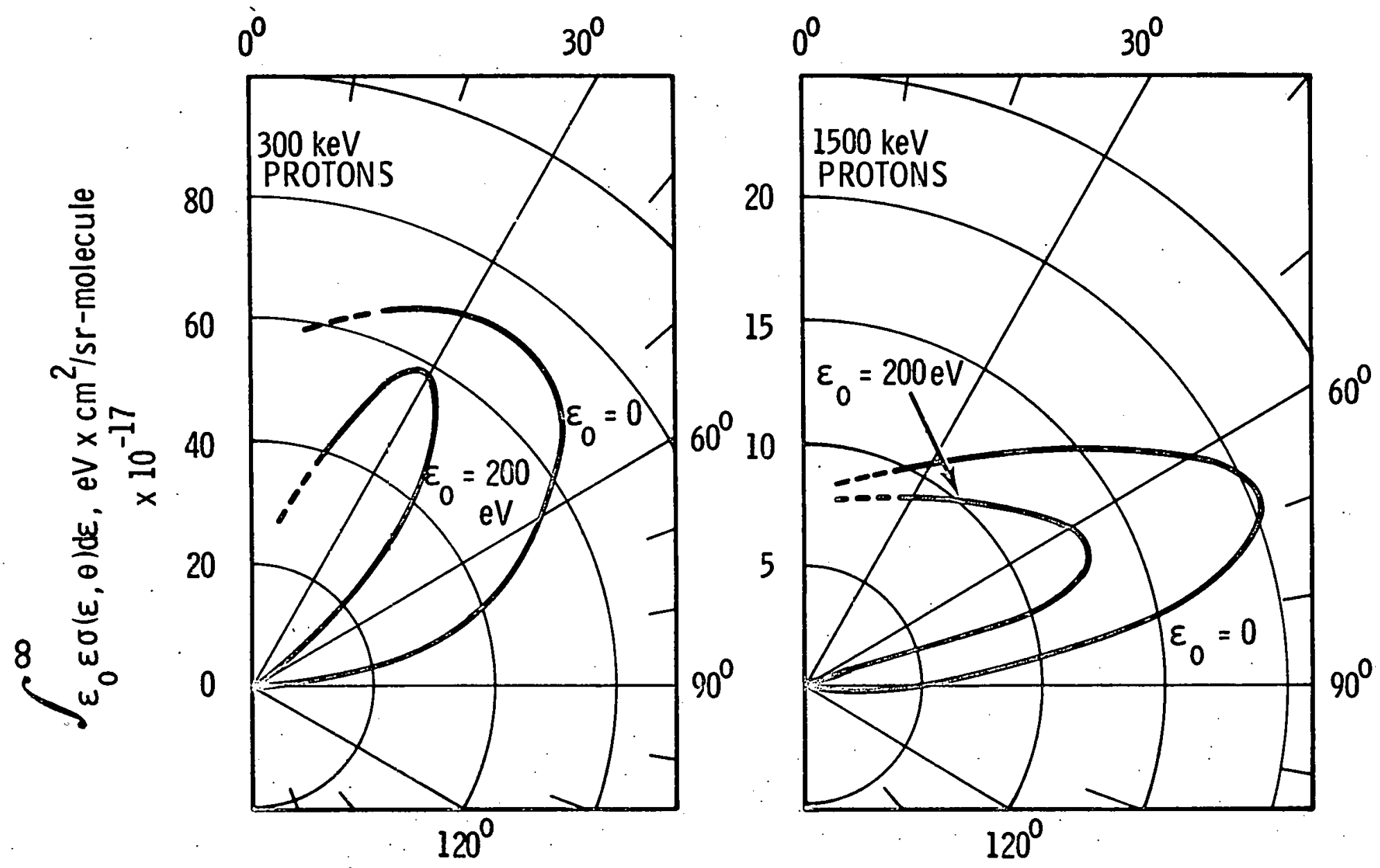

Fig. 4 

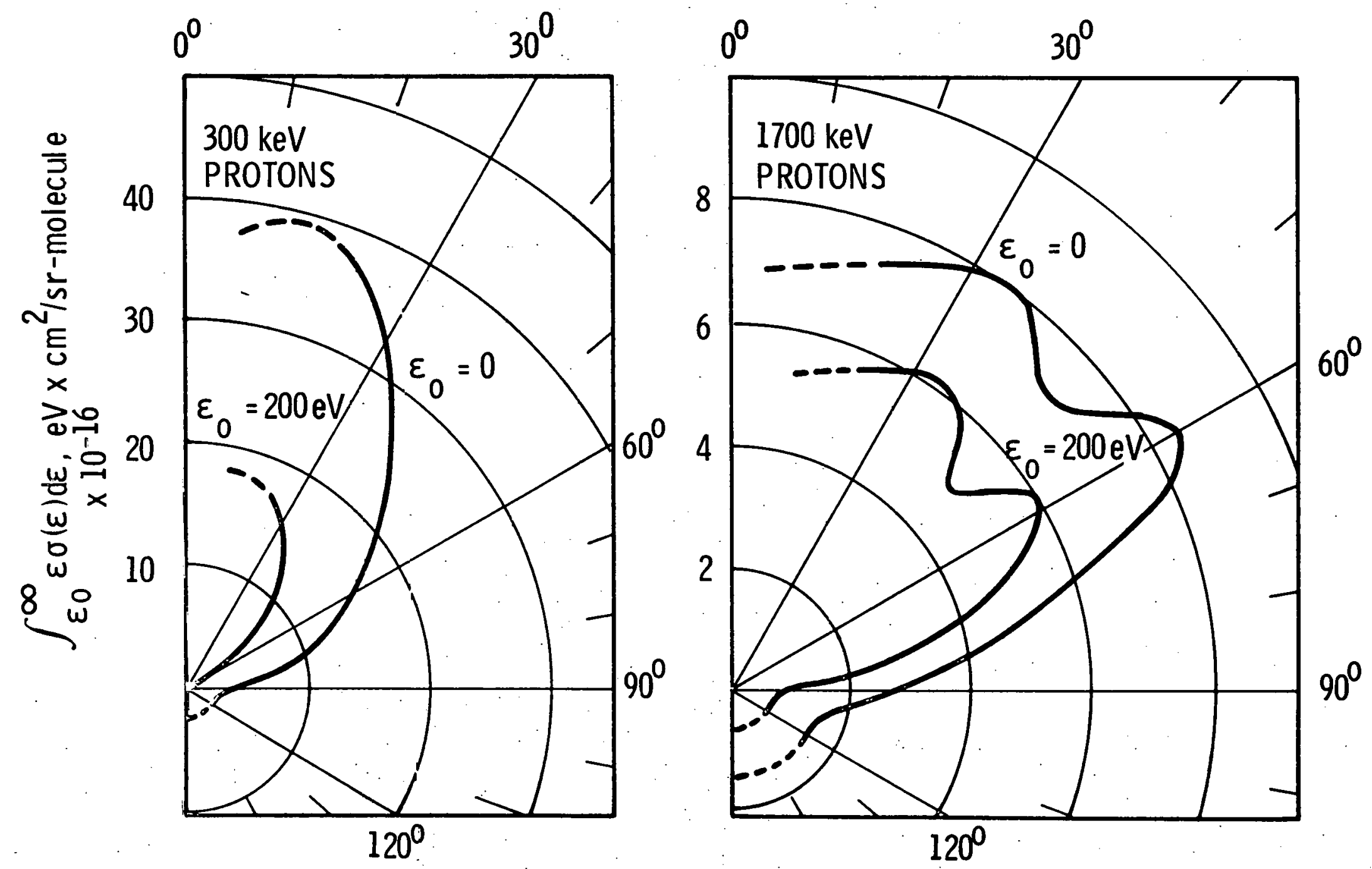

Fig. 5 


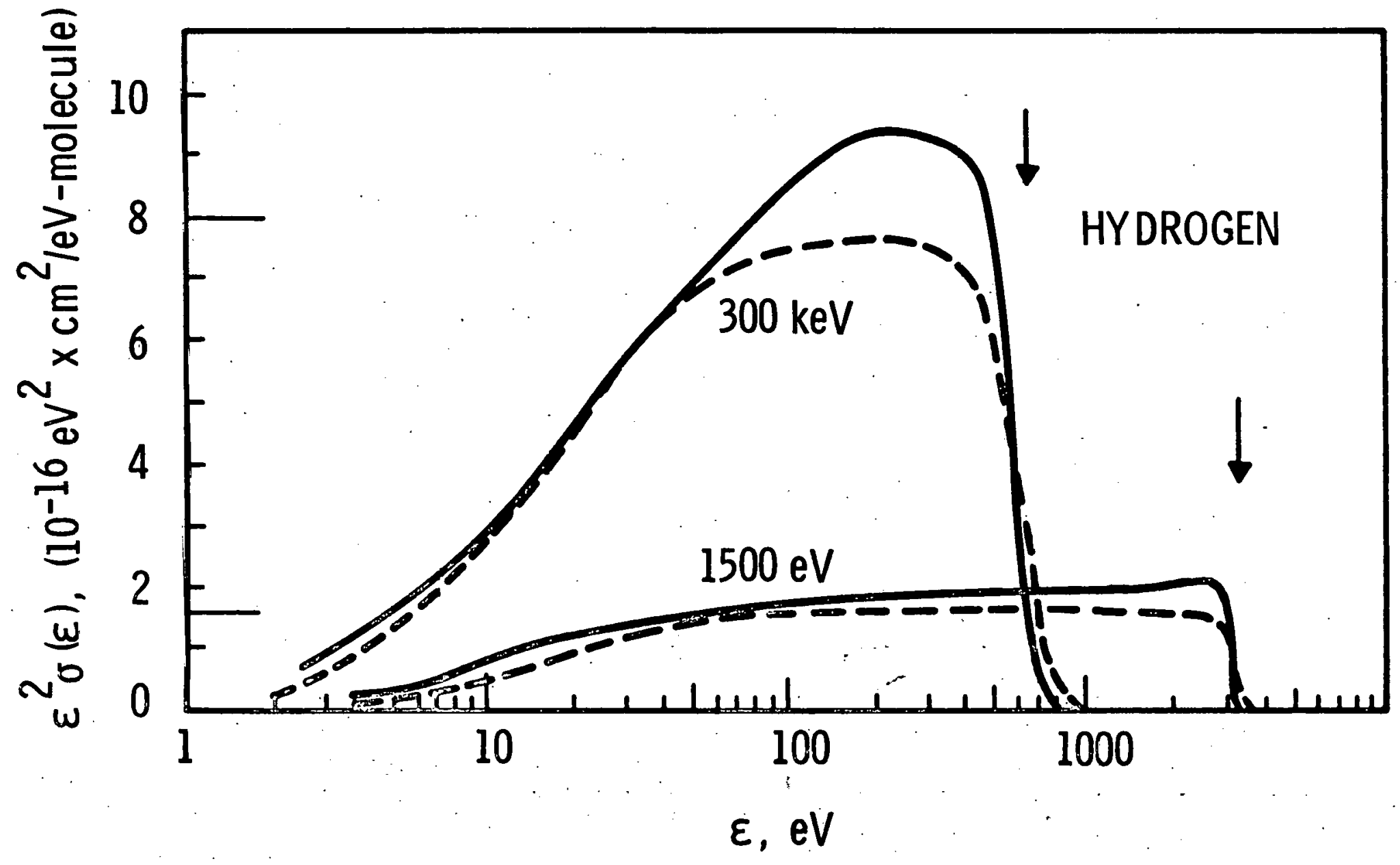

Fig. 6 


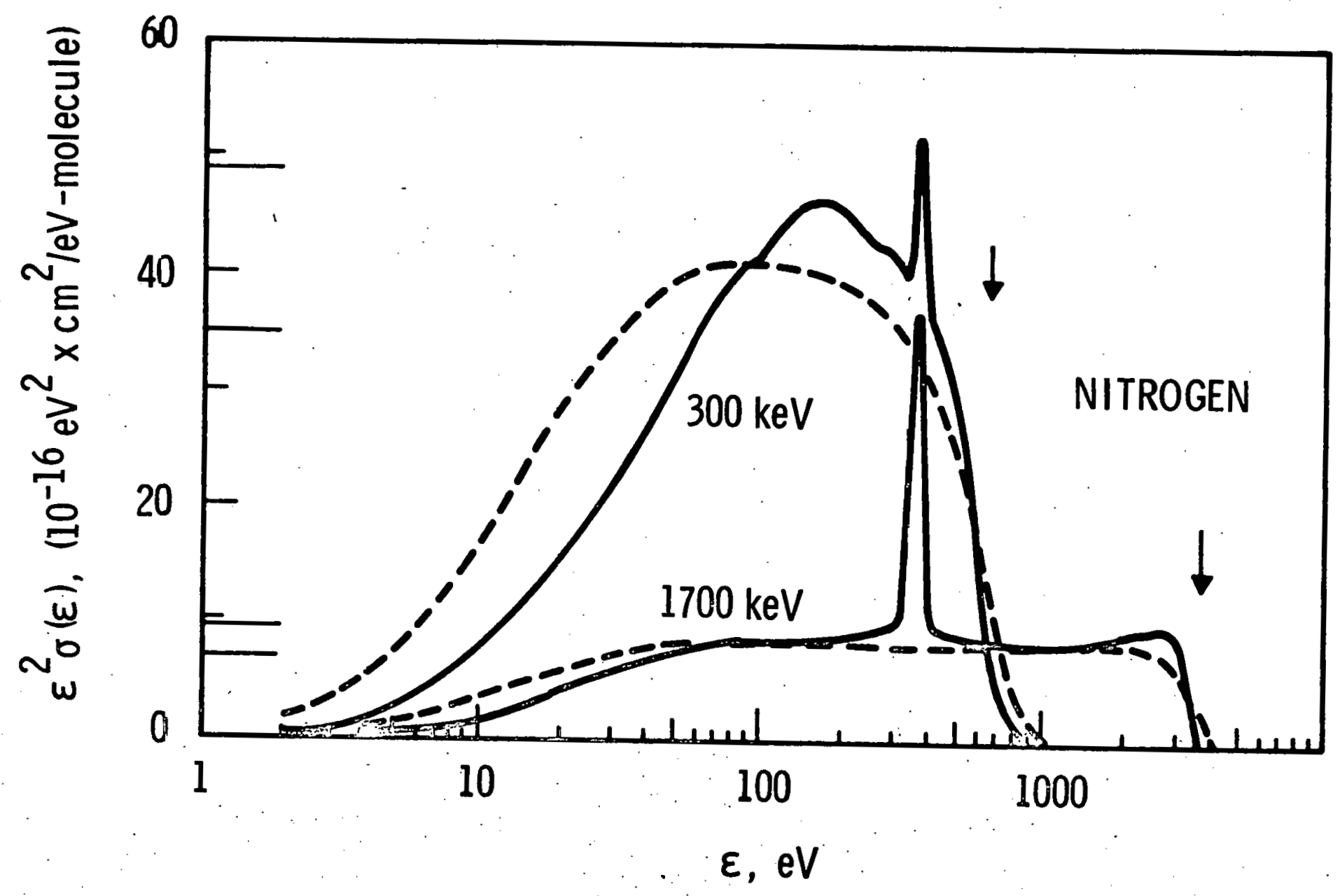

Fig. 7 

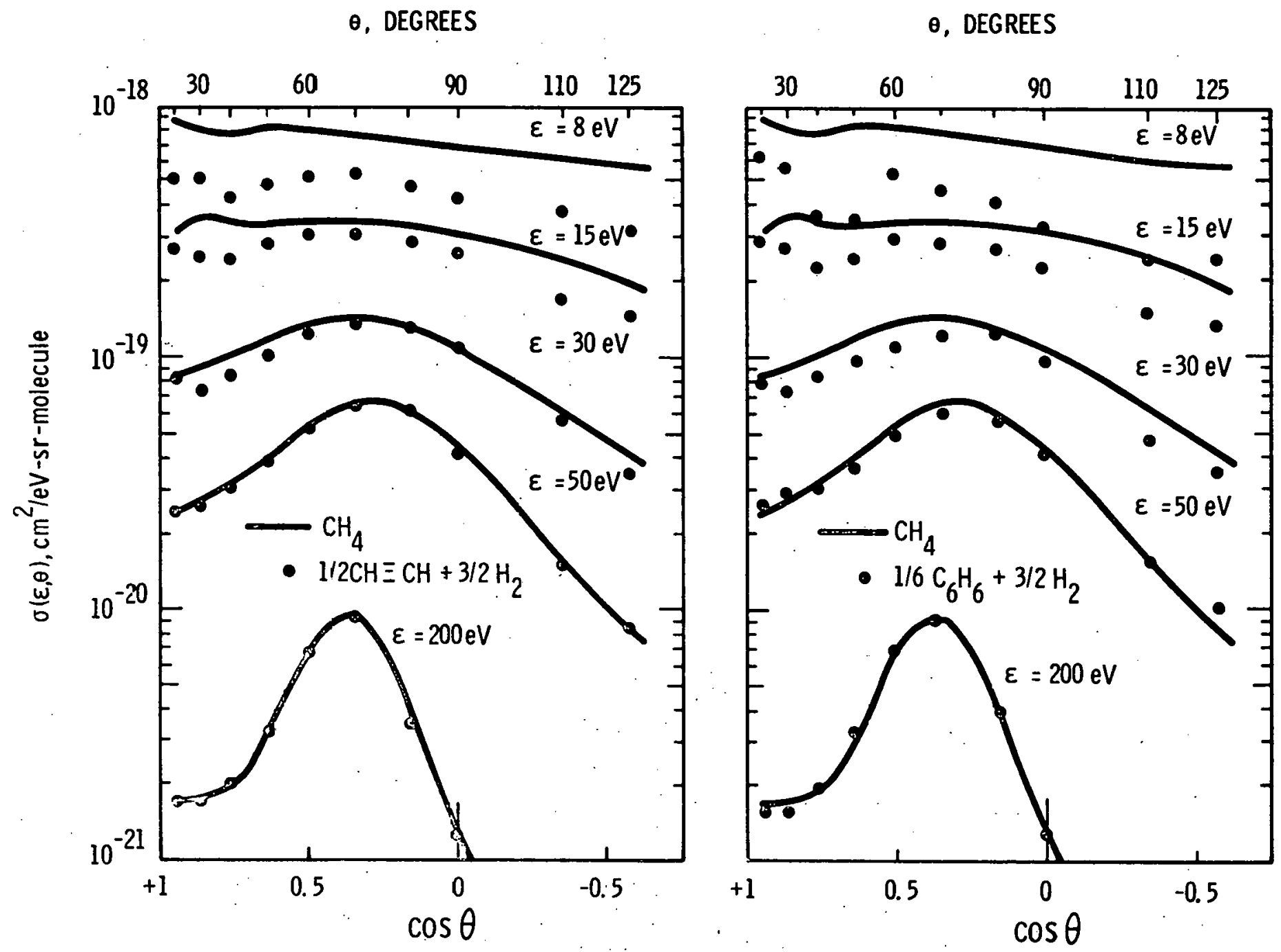

Fig. 8 


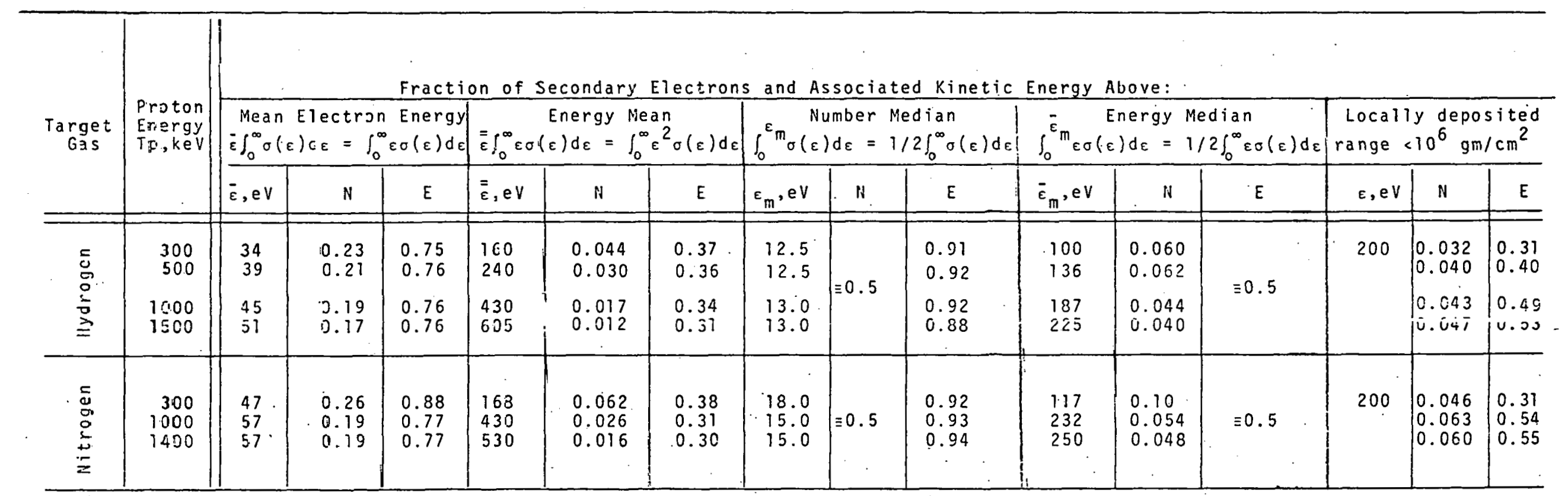

